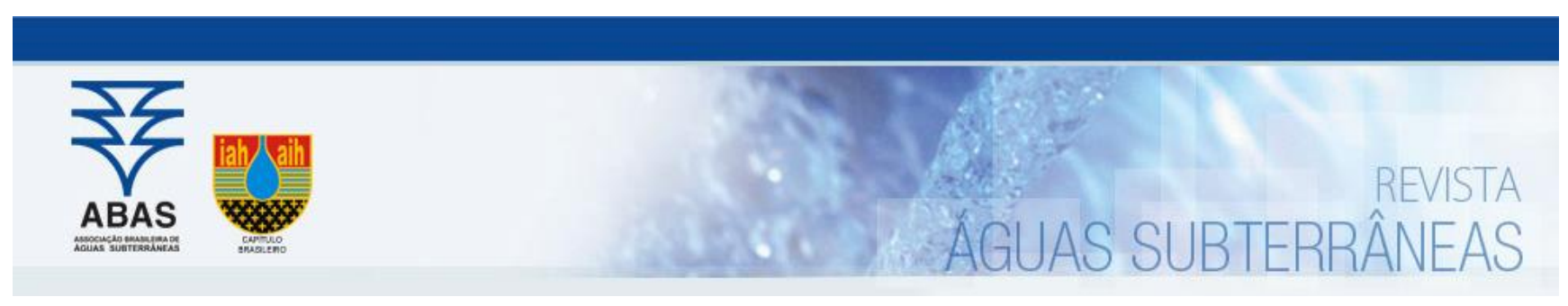

\title{
Artigos
}

\section{Salinización de suelos de textura fina por ascenso capilar a partir del acuífero freático hipersalino somero (Bahía Blanca,} Argentina)

\author{
Salinização de solos de textura fina por ascensão capilar a partir do \\ aquífero raso hipersalino (Bahía Blanca, Argentina)
}

\author{
Leonardo Ezequiel Scherger ${ }^{1,2}$; Claudio Lexow ${ }^{1}$; Victoria Zanello² ; Micaela Carbajo Castoldi ${ }^{\bowtie}$ \\ 1 Departamento de Geología, Universidad Nacional del Sur (UNS), Bahía Blanca, Buenos Aires, Argentina. \\ ${ }^{2}$ Centro de Geología Aplicada, Agua y Medio Ambiente (CGAMA-CIC-UNS). Comisión de Investigaciones Científicas, Bahía \\ Blanca, Argentina. \\ ${ }^{3}$ CONICET, CCT Bahía Blanca. Camino La Carrindanga, Bahía Blanca, Argentina.
}

\leonardo.scherger@uns.edu.ar, lexow@uns.edu.ar, zanellovictoria1992@gmail.com, micaela.carbajo@hotmail.es

\begin{tabular}{|c|c|}
\hline & Resumem \\
\hline $\begin{array}{l}\text { Palabras-chave: } \\
\text { Zona no saturada. } \\
\text { Salinidad, } \\
\text { Hydrus. } \\
\text { Bahía Blanca. }\end{array}$ & $\begin{array}{l}\text { El sector sudoeste de la Ciudad de Bahía Blanca, Argentina se caracteriza por la presencia de suelos de textura fina y } \\
\text { de un acuífero freático hipersalino somero, condiciones que generan la acumulación de sales en los niveles superficia- } \\
\text { les del suelo. Este trabajo tiene como objetivo determinar los principales mecanismos de movilidad y acumulación de } \\
\text { sales en un terreno limo arcilloso mediante la aplicación del código HYDRUS 2D/3D. El movimiento de sales en el perfil } \\
\text { de suelo se encuentra ligado a la hidrodinámica natural de la zona no saturada, donde los procesos de capilaridad y } \\
\text { evapotranspiración son los principales agentes de acumulación de solutos. Los periodos de mayor acumulación cor- } \\
\text { responden a los meses cálidos y secos, de alta demanda de humedad por la atmósfera. En estas condiciones se } \\
\text { generan concentraciones de sales en los niveles superficiales del suelo de hasta } 18000 \text { mg. L-1. Las precipitaciones } \\
\text { permiten el ingreso de agua de baja salinidad al perfil de suelo, ocasionando los procesos de lavado y dilución. Las } \\
\text { sales son transportadas hacia los niveles inferiores de la zona no saturada, efecto visible en años pluviométricamente } \\
\text { húmedos. Las concentraciones simuladas en la franja capilar se mantuvieron en el rango de entre } 3000 \text { mg. } \mathrm{L}^{-1} \text { y } \\
10000 \text { mg.L-1. Conocer el proceso de salinización de suelos en áreas urbanas, será fundamental para mitigar las con- } \\
\text { secuencias de las zonas afectadas. }\end{array}$ \\
\hline
\end{tabular}

\section{Resumo}

Palavras chave:

Zona não saturada.

Salinidade.

Hydrus.

Bahía Blanca.

O setor sudoeste da cidade de Bahia Blanca, Argentina é caracterizado pela presença de solos de textura fina e um aquífero hipersalino raso, condições que causam a acumulação de sais nos níveis superficiais do solo. Este estudo tem por objetivo determinar os principais mecanismos de mobilidade e acumulação de sais em um solo limo-argiloso, aplicando o software HYDRUS 2D/3D 0 movimento de sais no perfil do solo está ligado à hidrodinâmica naturais da zona não saturada, onde os processos de capilaridade e evapotranspiração são o principal acumulador de agentes solutos. Os períodos de maior acumulação correspondem aos meses quentes e secos, com alta demanda de umidade na atmosfera. Nestas condições, concentrações salinas são geradas nos níveis superficiais do solo de até 18.000 mg.L-1. A precipitação pluviométrica permite o ingresso de água de baixa salinidade ao perfil do solo, causando os processos de lavagem e diluição. Os sais são transportados para os níveis mais baixos da zona não saturada, efeito visível em anos pluviométricamente húmidos. As concentrações simuladas na franja capilar manteve-se na gama de entre 3000 mg.L-1 e 10000 mg.L-1. Conhecer o processo de salinização do solo em áreas urbanas é fundamental para mitigar as consequências das áreas afetadas.

\section{Abstract}

The Bahía Blanca City southwest sector, in Argentina, is characterize by the presence of fine texture soil and a hypersaline phreatic shallow aquifer, conditions that generate the accumulation of salts in the soil superficial levels. The purpose of this paper is to determinate the main salts mobility and accumulation mechanisms in a silt and clayey ground through the application of the HYDRUS 2D/3D code. The salt movements in the soil outline is linked to the natural hydrodynamics of the unsaturated zone, where the capillarity and evapotranspiration processes are the main agents of solutes accumulation. The periods of greatest accumulation correspond to the warm and dry months, with a high demand for humidity in the atmosphere. Under these conditions, salt concentrations are generated in the soil surface levels of up to $18,000 \mathrm{mg}$. $\mathrm{L}^{-1}$. The precipitations allow the entrance of low salinity water into the soil profile, causing the processes of washing and dilution. The salts are transported to the lower levels of the unsaturated zone, a visible effect in pluviometrically wet years. The concentrations simulated in the capillary fringe maintain in the range between 3,000 mg. L-1 and 10,000 mg. $\mathrm{L}^{-1}$. Knowing the salinization process in urban areas soils would be fundamental to mitígate consequences in affected areas. 


\section{INTRODUCCIÓN}

La salinización representa uno de los principales procesos para la pérdida de calidad del suelo, donde generalmente sus consecuencias son referidas a zonas agrícolas-ganaderas. Sin embargo esta problemática no debe ser descartada en áreas urbanas, donde las sales presentes en la zona no saturada pueden generar deterioros considerables sobre las estructuras de hormigón y pavimentos (GRIZINIK y HIRTZ, 2000). E ingreso de sales al perfil de suelo suele presentar en general dos orígenes bien definidos: producto del riego de aguas con altos contenidos de iones disueltos o provenientes desde un acuífero hipersalino por el proceso de capilaridad. En este último caso los efectos son magnificados en presencia de un nivel freático somero, que se encuentre a escasos metros de la superficie.

El trabajo se centra en el sector sudoeste de la ciudad de Bahía Blanca, Argentina, correspondiente a la franja costera aledaña al estuario de nombre homónimo. Este sector se caracteriza por la presencia de suelos de texturas finas y de un nivel freático somero, condiciones que determinan el predominio de movimientos verticales y la transferencia del agua por ascenso capilar a través de la zona no saturada, propiciando la concentración de sales por evapotranspiración (PÉREZ MARFIL et al., 2017). Tanto la evaporación como la transpiración remueven la humedad del suelo, generando un incremento de las concentraciones salinas en el agua edáfica remanente (CORWIN et al., 2007).

En diversos sectores de la localidad se han detectado grietas y fisuras en los pavimentos y estructuras de hormigón que, relacionadas a las condiciones del subsuelo que conforma el área, podrían estar vinculadas a este proceso (LEXOW et al., 2017). En sectores urbanos la interacción humana, dado por urbanización sobreimpuesta, alteraciones en la red de drenaje natural, infiltración de aguas al subsuelo, etc; genera condiciones que aceleran los procesos de salinización (GRIZINIK y HIRTZ, 2000).

Los modelos de simulación han sido herramientas muy valiosas para estudios que involucran procesos complejos e interactivos en el flujo de agua y transportes de solutos dentro de la zona no saturada. El código HYDRUS 2D/3D (Simunek et al., 2006) permite la simulación del flujo de agua tanto en condiciones saturadas como no saturadas según la resolución de la ecuación de Richards y el transporte de solutos mediante la resolución de la ecuación de advección-dispersión. El objetivo de este trabajo es analizar los procesos que generan la salinización de suelos limo-arcillosos por ascenso capilar de sales desde el acuífero freático somero. Las simulaciones en el software HYDRUS 2D/3D permitirán: la modelación del flujo de agua y sales asociadas en la zona no saturada bajo gradientes hidrodinámicos naturales, evaluar la magnitud del aporte de sales al suelo y verificar la situación actual, mediante la comparación con datos de campo. Conocer el proceso de salinización de suelos en áreas urbanas, será fundamental para mitigar las consecuencias de las zonas afectadas, soluciones que podrán hacerse extensivas a regiones con problemáticas similares.

\subsection{Características generales del área de estúdio}

La localidad de Bahía Blanca se encuentra en el sector Sudoeste de la Provincia de Buenos Aires, Argentina aproximadamente en los $38^{\circ} 43^{\prime \prime}$ Latitud Sur y $62^{\circ} 16^{\prime \prime}$ Longitud Oeste (Fig. 1). El área de estudio se ubica en el sector Sudoeste de la localidad, en cercanías a la localidad de Ingeniero White, hacia el Oeste y al estuario de Bahía Blanca, hacia el Sur, abarcando un área aproximada de $12 \mathrm{~km}^{2}$.

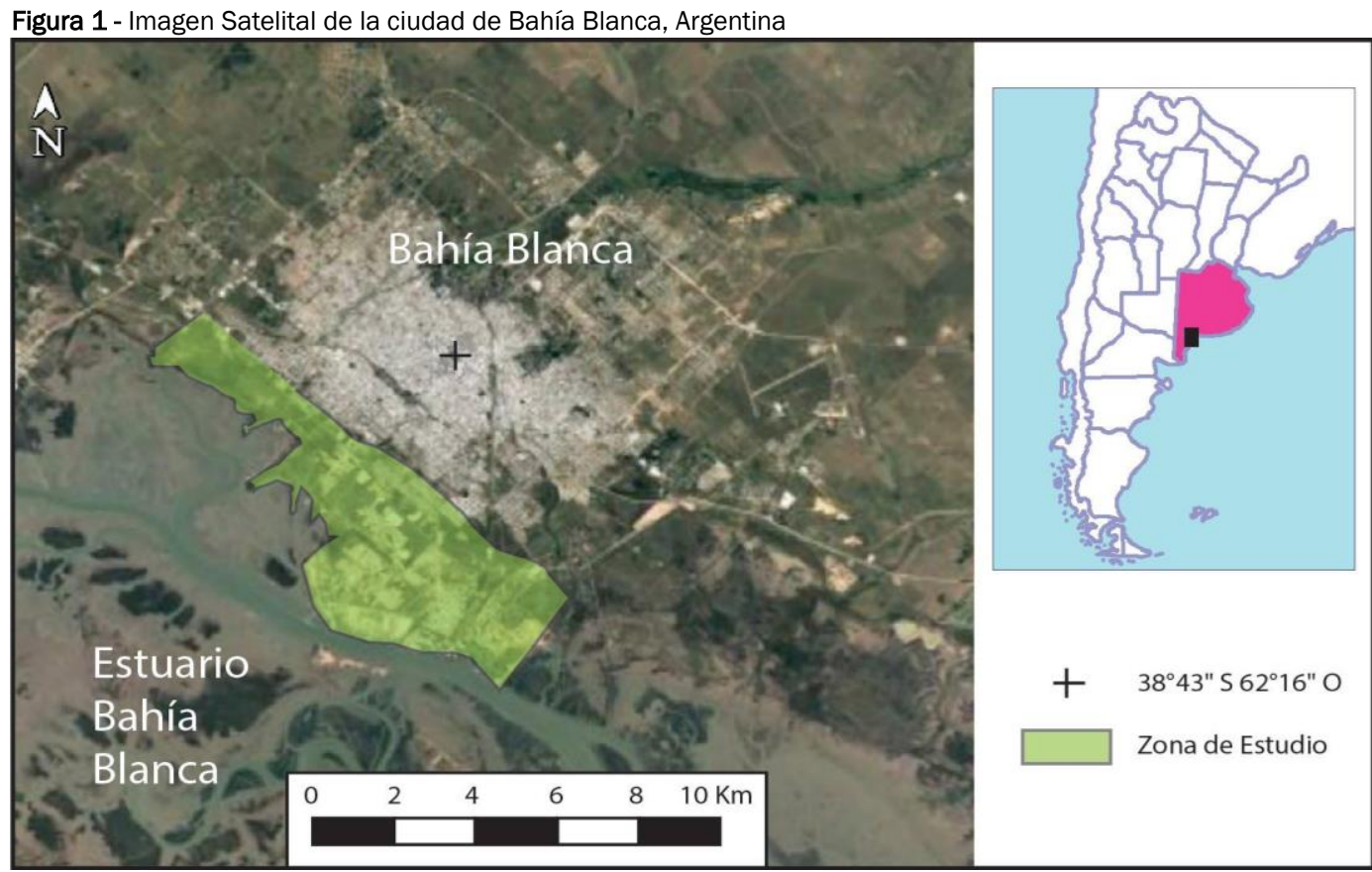

Geomorfológicamente, el área comprende una plataforma de 
abrasión marina sobre la cual se depositan sedimentos associados a rellenos de marea. Las áreas que denotan condiciones naturales se presentan como relictos restringidos, ya que la mayor parte del área se encuentra modificada debido a la introducción de material de relleno, utilizado para dar sustento a las construcciones y redes viales. El subsuelo se compone por los Sedimentos Pampeanos, limos loessicos de edad Pliocena, que subyacen a los depósitos marinos recientes de la Formación Maldonado (FIDALGO, 1983). Esta última consiste en una facies granodecreciente, con arenas en la base que pasan gradualmente a limos arenosos, limos arcillosos y arcillas limosas. El acuífero costero libre se encuentra contenido por la Formación Maldonado conjuntamente con los Sedimentos Pampeanos. Aunque existen diferencias en sus características litológicas, ambas pueden ser consideradas como una única unidad hidrogeológica. El acuífero es catalogado como hipersalino con valores de salinidad de entre 5000 mg. $\mathrm{L}^{-1}$ a $50000 \mathrm{mg} \cdot \mathrm{L}^{-1}$, hacia la zona de descarga, siendo el agua subterránea clasificada como clorurada sódica a clorurada sulfatada sódica (BONORINO y SALA, 1983). El nivel freático mantiene profundidades someras, cercanas al metro en la mayor parte del año, formando anegaciones temporales durante los periodos húmedos de máxima expresión.

Los suelos naturales presentan texturas franco limosas a franco arcillosas, con escasa cobertura vegetal, en general de tipo halófita. En superficie, muestran efluorescencias salinas y en profundidad grandes cantidades de carbonato de calcio y yeso, clasificándolos como Acuisalid típico de acuerdo a la Soil Taxonomy (USDA, 2003).

\section{METOdOLOGÍA}

\subsection{Recolección de datos}

En campo se relevó la profundidad del nivel freático y se tomaron muestras de suelo y agua subterránea para su posterior análisis en laboratorio y caracterización físico-química. Las muestras de suelo fueron recolectadas de Abril a Agosto de 2017 mediante pala helicoidal, en intervalos de $30 \mathrm{~cm}$, hasta la profundidad donde se interceptó el nivel freático. Las muestras de agua fueron tomadas mediante muestreador tipo "bailer" desde el pozo cavado. Los ensayos sobre muestra de suelo fueron realizados en el Laboratorio de Análisis Químicos (LANAQUI), CERZOS-CONICET-UNS. Sobre el extracto de saturación del suelo se determinaron: conductividad eléctrica, cloruros y sulfatos. El agua subterránea fue analizada en el Departamento de Bromatología y Protección de la Salud (Municipalidad de Bahía Blanca), determinándose: conductividad eléctrica, total de sólidos disueltos (TSD) e iones mayoritarios. En la tabla 1 se indican los valores medios correspondientes a muestras de suelo y agua.

Tabla 1 - Valores medios correspondientes a los análisis químicos realizados sobre muestras de suelo y agua subterránea.

\begin{tabular}{|c|c|c|c|c|c|}
\hline \multicolumn{6}{|c|}{ Agua Subterránea } \\
\hline C.E $(\mu S / \mathrm{cm})$ & $\begin{array}{l}\text { T.D.S } \\
\text { (mg/L) }\end{array}$ & p.H & Cloruros (mg/L) & $\begin{array}{l}\text { Sulfatos } \\
\text { (mg/L) }\end{array}$ & Dureza total $\mathrm{CaCO} 3(\mathrm{mg} / \mathrm{L})$ \\
\hline 9780 & 5744 & 9 & 1353 & 1354 & 594 \\
\hline \multicolumn{6}{|c|}{ Suelo } \\
\hline Profundidad & & C.E extracto $(\mu S / \mathrm{cm})$ & & uros $(\mathrm{mg} / \mathrm{L})$ & Sulfatos $(\mathrm{mg} / \mathrm{L})$ \\
\hline $30 \mathrm{~cm}$ & & 4200 & & 780 & 436 \\
\hline $60 \mathrm{~cm}$ & & 4900 & & 1117 & 719 \\
\hline $90 \mathrm{~cm}$ & & 5100 & & 942 & 709 \\
\hline
\end{tabular}

Leyenda: C.E: conductividad eléctrica; T.S.D.: total de sólidos disueltos

\subsection{Elaboración del Modelo}

El modelo de transporte de sales solubles en el perfil no saturado se realizó mediante la aplicación del software HYDRUS 2D/3D (SIMUNEK et al., 2006). El código HYDRUS ejecutable en ambiente Windows, resuelve la ecuación de Richards modificada (1) para el flujo no saturado y de advección-dispersión (2) para el transporte de solutos, en determinada sección de estudio, según condiciones iniciales y de borde establecidas por el usuario. La ecuación de Richards modificada, que adhiere un término sumidero para incorporar la extracción de agua por raíces, es resuelta mediante el método de elementos finitos.

$\frac{\partial \theta}{\partial t}=\frac{\partial}{\partial x_{i}}\left[K\left(K_{i j}^{A} \frac{\partial h}{\partial x_{j}}+K_{i z}^{A}\right)\right]-S$

Donde $\theta$ representa la humedad volumétrica $\left(\mathrm{cm}^{3} \mathrm{~cm}^{-3}\right), \mathrm{h}$ es la presión matricial $(\mathrm{cm}), \mathrm{S}$ es el termino sumidero (cm.día-1), $x_{i}(i=1,2, \ldots, n)$ son las coordenadas espaciales, $t$ es el tiempo (s), $K_{i z}^{A}$ son las componentes del tensor de anisotropía adimensional y $\mathrm{K}$ es la conductividad hidráulica no saturada (cm.día1).

$$
\frac{\partial \theta(h)}{\delta t}+\frac{\delta(\theta c)}{\delta t}=\frac{\delta}{\delta z}\left(\theta D \frac{\delta c}{\delta z}-q c\right)-S
$$

Donde c representa la concentración en la fase liquida (mg.L1), D es el coeficiente de dispersión hidrodinámica $\left(\mathrm{cm}^{2} \cdot\right.$ día-1$\left.^{-1}\right)$, q representa el flujo no saturado $\left(\mathrm{cm}^{3}\right.$.día $\left.{ }^{-1}\right)$ y $\mathrm{z}(\mathrm{cm})$ es la dirección del flujo.

El periodo de simulación consistió en 2451 días, iniciando el 01/01/2011 y finalizando el 13/09/2017. El modelo hidráulico elegido para la simulación fue el propuesto por Van Genuchten-Mualem (3) (Van Genuchten, 1980).

$$
\mathrm{Se}=1+\left[(\alpha \mathrm{h})^{\mathrm{n}}\right]^{-\mathrm{m}}
$$


Donde $S_{e}=\left(\theta-\theta_{r}\right) /\left(\theta_{s}-\theta_{r}\right)$ es la humedad efectiva, $\alpha=h_{a}-1$ es un parámetro empírico determinado por la inversa del valor de entrada de aire, $\mathrm{h}$ corresponde a la tensión matricial y $\mathrm{n}$ y $\mathrm{m}$ son parámetros empíricos siendo $m=1-1 / n$. La conductividad hidráulica (4) se estima mediante la ecuación:

$$
\mathrm{K}(\mathrm{Se})=\mathrm{KsSe}^{\mathrm{l}}\left[1-\left(1-\mathrm{Se}^{\frac{1}{\mathrm{~m}}}\right)^{\mathrm{m}}\right]^{2}
$$

Donde $\mathrm{K}_{\mathrm{s}}$ corresponde a la conductividad hidráulica saturada y I es la conductividad de poro estimado en 0,5, como valor promedio de varios suelos por Mualem (1976).
Como dominio se definió un polígono rectangular de $120 \mathrm{~cm}$ de alto y $100 \mathrm{~cm}$ de ancho (Fig. 2a), representativo de la sección perpendicular del suelo, el cual fue discretizado en una malla de 801 nodos y 1512 subdominios triangulares. El material considerado consistió en un limo arcilloso cuyos parámetros hidráulicos (Tabla 2) fueron estimados mediante la función de pseudotransferencia Rosseta Lite (V1.1) (Schapp et al., 2001) a partir de la textura y densidad aparente del suelo.

Tabla 2 - Parámetros hidráulicos utilizados en la simulación

\begin{tabular}{|c|c|c|c|c|c|c|}
\hline Textura & $\delta_{a}$ & $\theta_{\mathrm{s}}\left(\mathrm{cm}^{3} \mathrm{~cm}^{-3}\right)$ & $\theta_{\mathrm{r}}\left(\mathrm{cm}^{3} \mathrm{~cm}^{-3}\right)$ & $\alpha$ & $n$ & $\mathrm{~K}_{\mathrm{s}}$ (cm.día-1) \\
\hline Limo arcilloso & 1,25 & 0,4795 & 0,0942 & 0,0113 & 1,4085 & 13,22 \\
\hline
\end{tabular}

Leyenda: $\delta_{a}$ : densidad aparente del suelo; $\theta_{\text {s: }}$ humedad saturada; $\theta_{\mathrm{r}}$ : humedad residual $\alpha$ y n: parámetros empíricos $\alpha=\mathrm{ha}^{-1}$; Ks: conductividad hidráulica saturada.

Las condiciones iniciales fueron introducidas en términos de presión matricial, considerando un valor de $-300 \mathrm{~cm}$ en superficie y un gradiente vertical en equilibrio hidráulico con la superficie inferior, cuyo valor es de $0 \mathrm{~cm}$ (Fig. 2b). El límite superior de la sección, superficie del suelo, se consideró dependiente de las condiciones atmosféricas. La evapotranspiración potencial de referencia fue estimada por medio del software CROPWAT 8.0 según el método de Penman-Monteith modificado según la FAO N 56 (1998). Se utilizaron valores medios mensuales de temperatura, humedad relativa, velocidad del viento y cantidad de horas de insolación para la serie climática 2011-2017 procedentes de la estación meteorológica del Co- mité Técnico Ejecutivo (CTE), de la localidad de Ing. White. En la figura 3, se informan las precipitaciones acumuladas expresadas en valores mensuales ingresadas al modelo.

Los límites laterales son nodos con ausencia de flujo, mientras que para el límite inferior se considera un potencial constante, $\mathrm{h}=0 \mathrm{~cm}$, representativo de un nivel freático estático. Aunque en la realidad, el nivel freático demuestra oscilaciones de entre $\pm 50 \mathrm{~cm}$ a lo largo del ciclo hidrológico, puede asumirse su posición a $120 \mathrm{~cm}$ de profundidad, valor medio del rango de oscilación.

Figura 2 - A. Gráfico esquemático de la sección estudio (2d) donde se detallan las condiciones de borde empleadas. B. Condiciones iniciales de tensión matricial y humedad volumétrica aplicadas al modelo.

A)

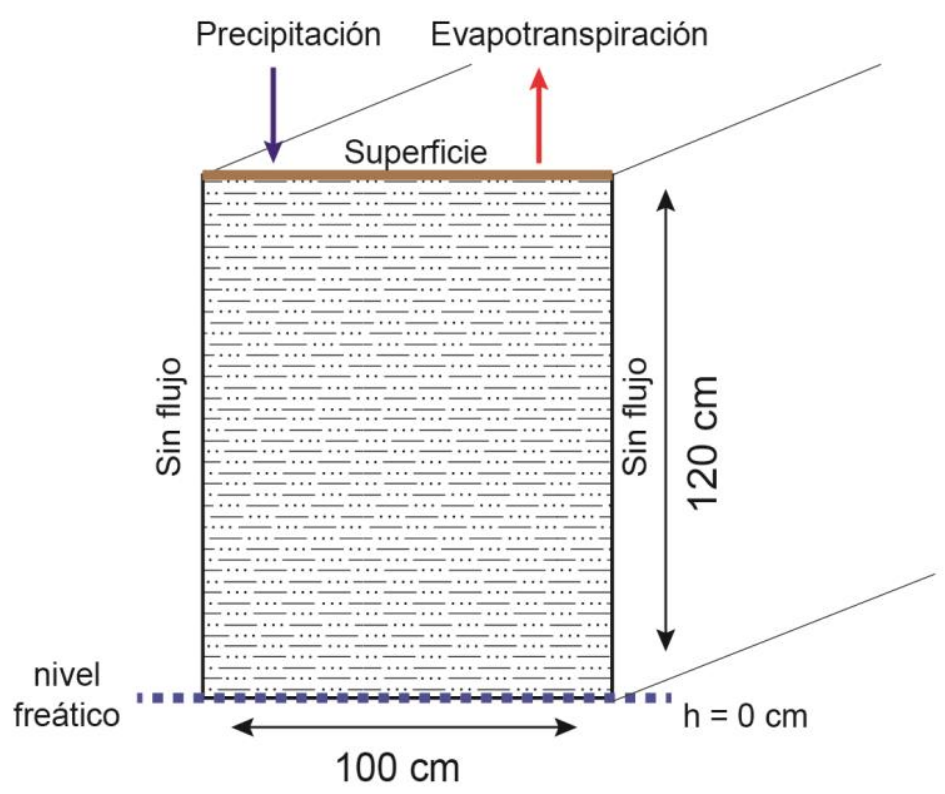

B)

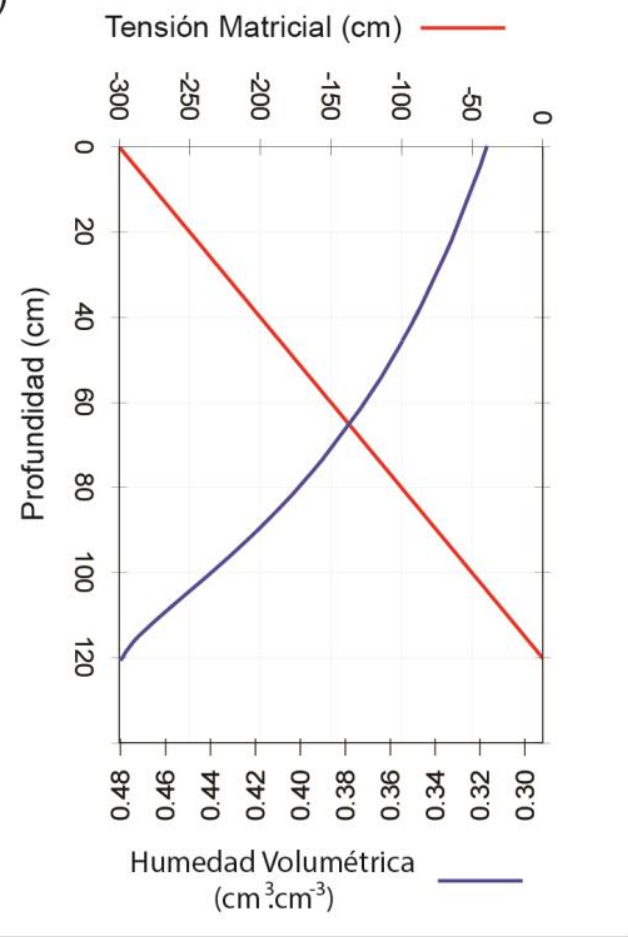


La extracción de agua por raíces (S) fue incorporada según el modelo de Feddes et al. (1978) (6).

$$
S=\alpha(h) S_{p}
$$

Donde $\alpha(\mathrm{h})$ representa la función de respuesta al stress hídrico $(0 \leq \alpha \leq 1)$ y $S_{p}$ indica el volumen potencial de extracción por las raíces de las plantas. El volumen extraído se asume cero en condiciones saturadas $\left(h_{1}\right)$ o cuando los potenciales mátricos son menores al punto de marchitez permanente $\left(h_{4}\right)$. Por su parte $\alpha$ toma el valor 1 , siendo así la máxima expresión de $\mathrm{S}=\mathrm{S}_{\mathrm{p}}$, cuando $\mathrm{h}_{2}<\mathrm{h}<\mathrm{h}_{3}$.Para el rango de presiones $h_{4}<h<h_{3} \circ h_{2}<h<h_{1}$, los volúmenes extraídos disminuyen linealmente a medida que disminuyen o aumentan respectivamente los potenciales de presión. Los parámetros utilizados corresponden a $\mathrm{h}_{1}=-10 \mathrm{~cm}, \mathrm{~h}_{2}=-25 \mathrm{~cm}, \mathrm{~h}_{3}=-200$ $\mathrm{cm}$ a $-800 \mathrm{~cm}$ y $h_{4}=-8000 \mathrm{~cm}$, tomados de Wesseling (1991) para pasturas de raíces someras.

Figura 3 - Grafico de lluvias acumuladas expresadas en valores mensuales. En línea punteada se indica el valor histórico para la zona (serie 1890 - 2000), tomado de Caló et al. (2004)

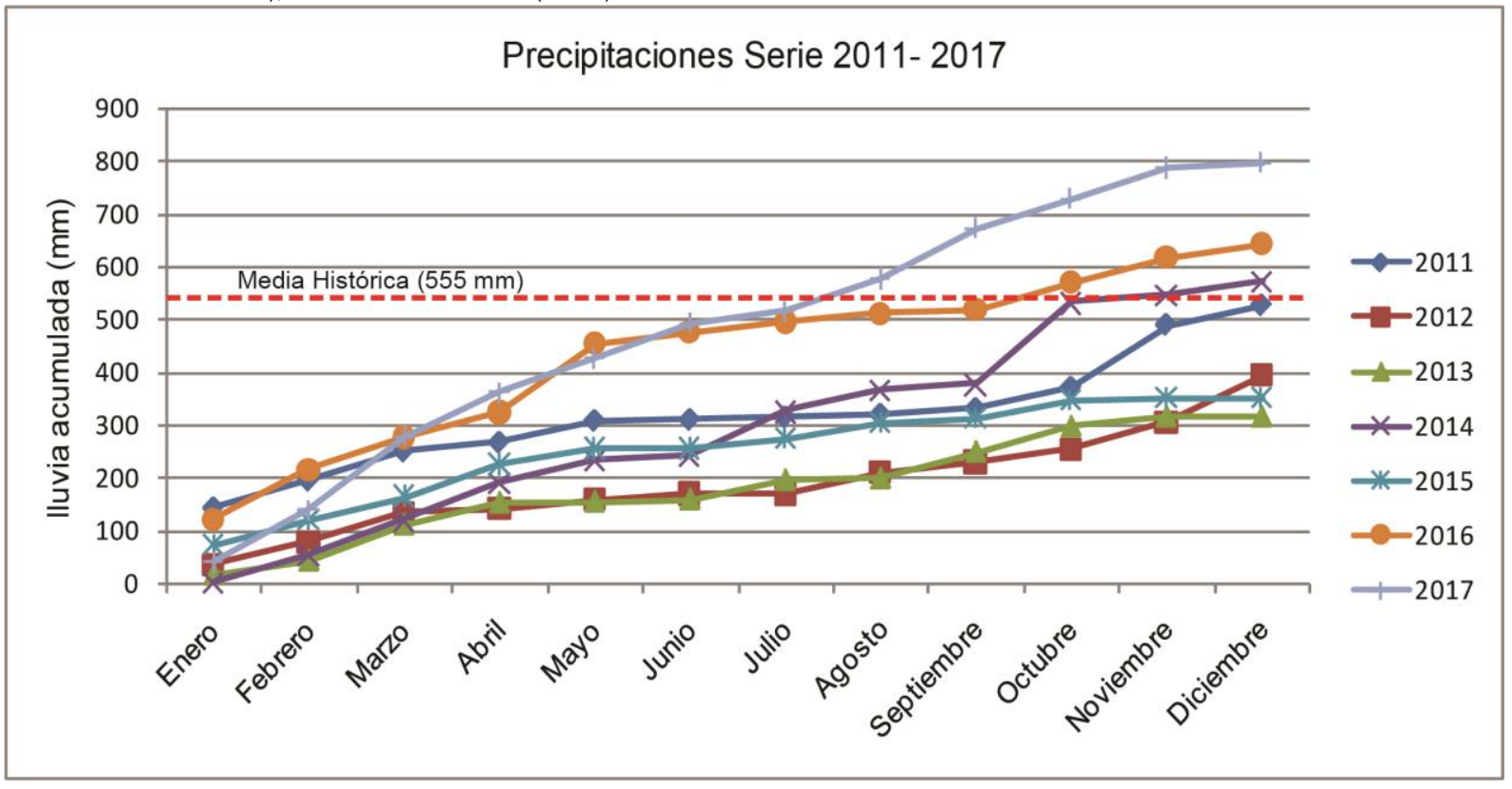

La concentración salina del agua subterránea (TSD) (7) se calculó a partir de la relación con la conductividad eléctrica (CE) la cual se obtuvo a partir del modelo matemático de estimación lineal para todas las muestras relevadas.

$\operatorname{TSD}\left(\frac{\mathrm{mg}}{\mathrm{L}}\right)=\operatorname{CE} * 0,638$

Asumiendo que la cantidad de sales adsorbidas en el complejo de cambio del suelo es despreciable, la concentración de sales en la solución edáfica (C) (8) se calculó mediante la relación entre TSD $\left(\mathrm{mg}_{\mathrm{cm}} \mathrm{cm}^{-3}\right)$ y la humedad volumétrica del suelo ( $\theta)\left(\mathrm{cm}^{3} \cdot \mathrm{cm}^{-3}\right)$.

$\mathrm{C}\left(\mathrm{mg} \cdot \mathrm{cm}^{-3}\right)=\frac{\text { TSD }\left(\mathrm{mg} \cdot \mathrm{cm}^{-3}\right)}{\theta\left(\mathrm{cm}^{3} \cdot \mathrm{cm}^{-3}\right)}$

La concentración de sales en el agua de lluvia se tomó como un valor constante de 64 mg.L-1 (Carrica, 1998). El transporte de sales se simuló considerando la ausencia de procesos reactivos (fuentes-sumidero) en el suelo y, tal lo formulan Simunek et al. (2006), se tomaron valores de dispersividad y difusividad, $17 \mathrm{~cm}$ y $5 \mathrm{~cm}^{2}$. día-1 respectivamente, a partir de una base de parámetros optimizados en la simulación de sales solubles en suelos similares (Forkutsa et al. 2009 y Phogat et al. 2013).

\section{RESULTADOS Y DISCUSIONES}

\subsection{Comportamiento Hidrodinámico}

Las simulaciones obtenidas reflejan la dinámica del sistema no saturado, donde las características del terreno limo arcilloso natural y las condiciones atmosféricas e hidrológicas del entorno son los factores que controlan los flujos hídricos verticales dentro de la zona no saturada.

El perfil de suelo refleja humedades comprendidas entre 0.38 $\mathrm{cm}^{3} . \mathrm{cm}^{-3}$ a $0.48 \mathrm{~cm}^{3} . \mathrm{cm}^{-3}$ para el periodo simulado, donde los contenidos máximo y mínimos de humedad son representados por los niveles superficiales del perfil (Fig. 4a). Los nodos de $15 \mathrm{~cm}$ y $30 \mathrm{~cm}$ presentan mayores variaciones del contenido volumétrico hídrico que los nodos inferiores $(60 \mathrm{~cm}$ y 90 $\mathrm{cm}$ ); sujeto a las variaciones climáticas en superficie (periodos húmedos y cálidos respectivamente). Las precipitaciones de gran cuantía generan condiciones de saturación parcial en la superficie del terreno, donde la infiltración, encargada de redistribuir el bulbo húmedo a lo largo del perfil, es limitada por los bajos valores de permeabilidad vertical del terreno. Durante los meses de invierno, caracterizados por valores bajos de evapotranspiración, suelen generarse encharcamientos en campo, que pueden extenderse varios días luego de transcurrida la lluvia. 
A medida que incrementa la profundidad decrece la influencia de las condiciones atmosféricas superficiales, siendo las variaciones de humedad más restringidas. En esta zona, la acción de la evapotranspiración disminuye notablemente, dado que las raíces no se extienden más de $25 \mathrm{~cm}$ de profundidad en campo, siendo el proceso de capilaridad el fenómeno que permite la existencia de flujos verticales ascendentes. Los nodos de $60 \mathrm{~cm}$ y $90 \mathrm{~cm}$ mantienen contenidos volumétricos entre 42 a $44 \mathrm{~cm}^{3} \mathrm{~cm}^{-3}$ y entre 45 a $48 \mathrm{~cm}^{3} \mathrm{~cm}^{-3}$ respectivamente (Fig. 4a). Estos nodos son influenciados directamente por la presencia del nivel freático somero, el cual se ubica a $120 \mathrm{~cm}$ de profundidad durante el periodo simulado.

Las tensiones matriciales simuladas durante el periodo (Fig. 4b) son características de materiales finos, donde los gradientes de potencial en el perfil no saturado son consistentes con bajas velocidades de transferencia de humedad. Para que existan grandes cambios en el contenido volumétrico son necesarios grandes variaciones de tensión matricial, hecho que se refleja en las bajas pendientes de la curva de retención de humedad para estos materiales. Los nodos más superficiales, ubicados en la zona de evapotranspiración muestran los valo- res de succión más negativos, de hasta $-150 \mathrm{~cm}$, reduciéndose hasta valores de $0 \mathrm{~cm}$ durante la saturación del terreno. En el sector de la franja capilar las tensiones se mantienen con valores menores que la presión de entrada de aire $\left(h_{a}\right)$, considerada en $-88,4 \mathrm{~cm}$ para el material limo arcilloso. Aquí la posición del nivel freático determina una influencia directa sobre la tensión matricial y el contenido de humedad del terreno, siendo ambos los reguladores del flujo hídrico vertical en el sector no saturado.

\subsection{Evaluación del flujo no saturado}

La dirección del flujo hídrico en la zona no saturada, considerando únicamente el sentido vertical, queda determinada por las variaciones del potencial hidrodinámico total, donde la humedad se desplaza desde las zonas de mayor a menor potencial. De este modo, la zona no saturada puede ser subdividida en sectores donde el agua puede moverse en forma ascendente, con dominio de potenciales mátricos y contribuir a la evapotranspiración o descendente, con dominio de potencial gravimétrico, como recarga en tránsito.

Figura 4 - Simulación para el periodo 2011-2017 para cada nodo de observación. a) Ө: humedad volumétrica. b) h: tensión matricial.

A)

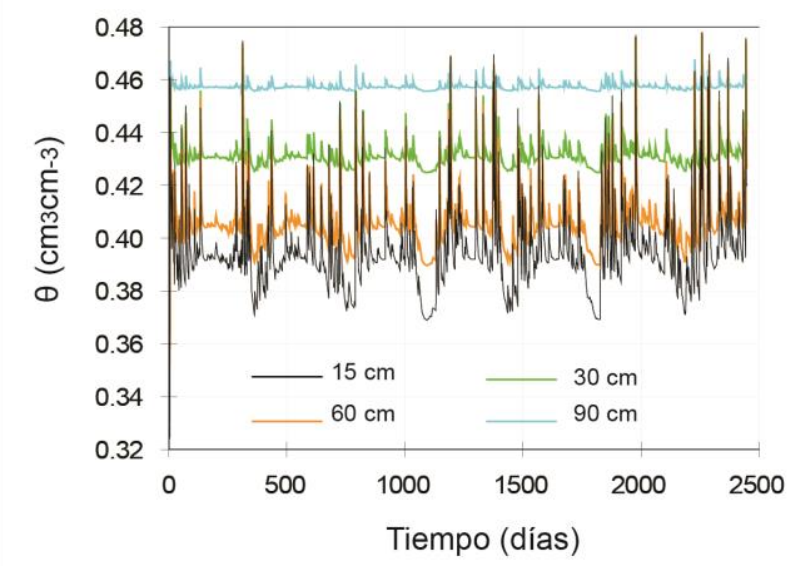

Las simulaciones del potencial hidrodinámico total poseen un registro muy diverso durante el periodo 2011- 2017, aunque pueden agruparse en cuatro escenarios, caracterizadas por diferentes situaciones de flujo, siendo los mismos netamente ascendente, netamente descendente, ascendente en la por-
B)

Tensión Matricial

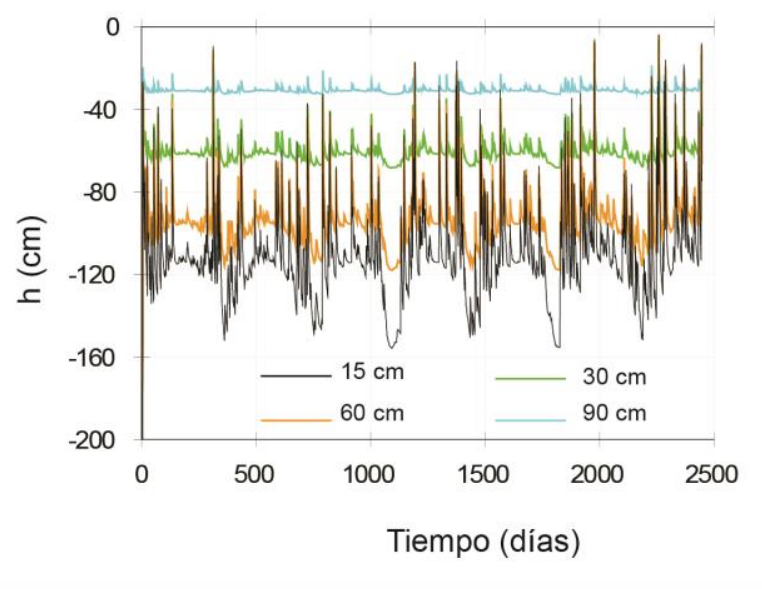

ción superior y descendente en la porción inferior o viceversa (Fig. 5). En estos dos últimos casos, se destaca la existencia de un plano de flujo cero, donde la humedad diverge o converge respectivamente. 
Figura 5 - Simulación del potencial hidrodinámico total para el periodo 2011-2017 para cada nodo de observación. En flechas se indica la condición de flujo para los periodos más relevantes. P.H.T: potencial hidrodinámico total

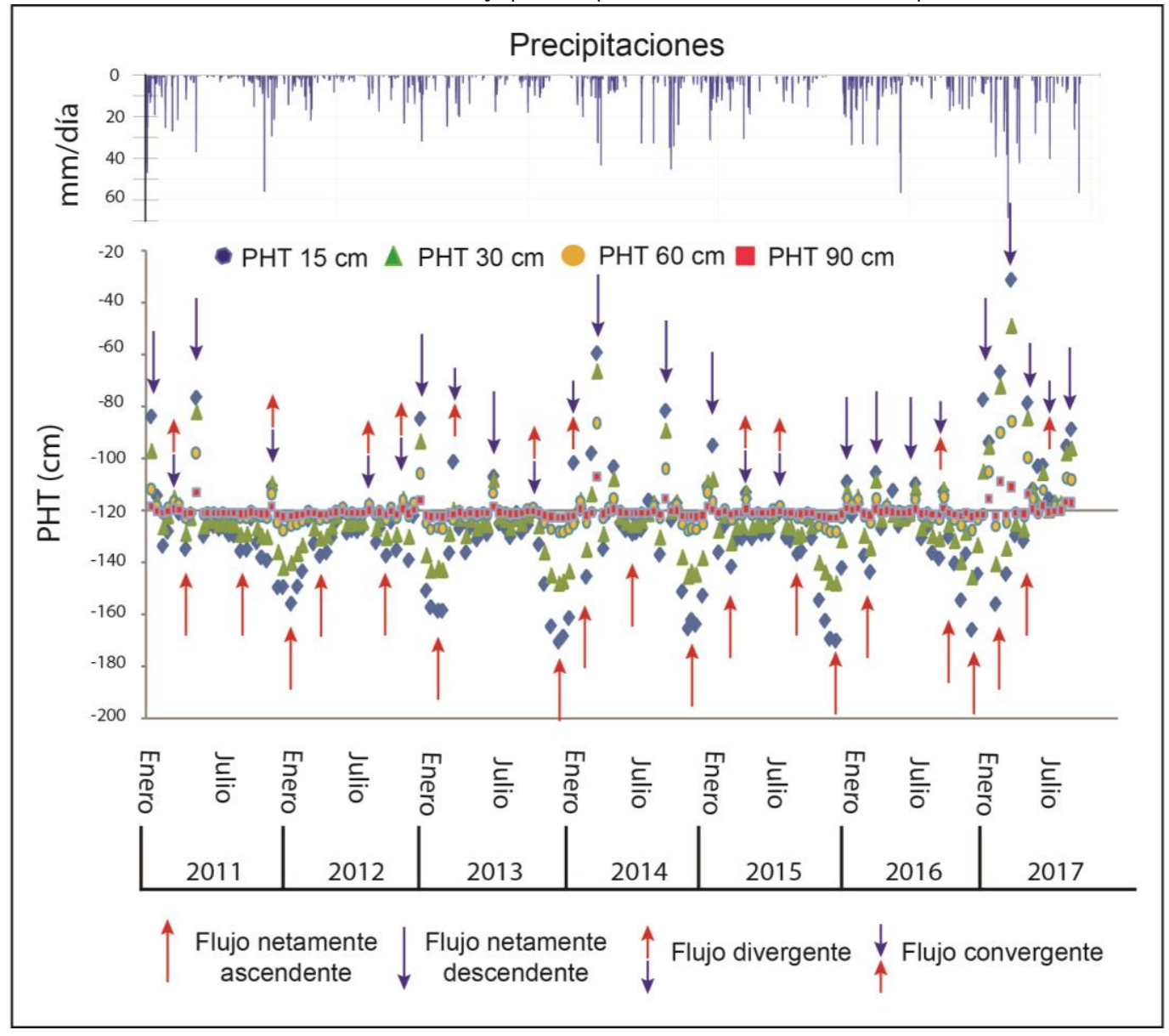

La existencia de flujo netamente ascendente en el perfil no saturado está asociada a periodos climáticos secos y cálidos, caracterizados por altos valores de evapotranspiración potencial. La cercanía del nivel freático a la superficie, permite el ascenso directo de humedad a los niveles superficiales del suelo, fenómeno promovido a su vez por las características texturales del terreno. Bajo estas condiciones, las sales solubles podrán ser movilizadas desde el acuífero hipersalino conjuntamente con el agua capilar, acumulándose en los niveles superficiales del suelo a medida que la humedad es transferida a la atmósfera.

Las precipitaciones permiten el ingreso de agua al perfil, generando un aumento de la humedad en los poros del terreno, disminuyendo así la tensión capilar. Cuando las fuerzas gravitatorias superan la componente matricial del potencial hidrodinámico total, se generan flujos verticales descendentes en los niveles superiores de la zona no saturada, conformándose un plano de flujo cero convergente. Precipitaciones mayores a los $2 \mathrm{~mm}$ diarios son capaces de generar un plano de flujo cero convergente cuya duración y profundidad de emplazamiento, presenta una relación directa con la intensidad de las lluvias y el estado de humedad antecedente del suelo. Si las precipitaciones son de gran cuantía se logran establecer con- diciones de flujo netamente descendente. La magnitud de las precipitaciones necesarias depende de la humedad antecedente de los niveles superficiales del suelo, ligada a las condiciones atmosféricas reinantes en superficie. En época estival, las lluvias necesarias se estiman en $30 \mathrm{~mm}$ diarios. Por su parte, durante los meses de invierno, precipitaciones de 15 $\mathrm{mm}$.día-1 son capaces de generar el proceso. El agua infiltra, percola y posteriormente recarga al acuífero hasta que el descenso de humedad en la porción superior, ya sea por transferencia hacia los sectores profundos o debido al desecamiento por evapotranspiración, permite nuevamente el aumento en la succión del terreno y el predominio de flujos ascendentes en la porción superior. Bajo estas condiciones se genera un plano de flujo cero divergente, cuya profundidad podrá variar hasta que se retorne a alguno de los escenarios previos.

En la Figura 6, se esquematiza el modelo simplificado de flujo para la zona no saturada. Cabe destacar que el pasaje de un escenario a otro es sumamente dinámico, siendo en la realidad muy variables las condiciones de flujo no saturado, regulado principalmente por las condiciones meteorológicas e hidrogeológicas del subsuelo (heterogeneidad textural, estructura, propiedades físico-químicas y biológicas). 
Figura 6 - Modelo esquemático del flujo de humedad unidireccional vertical en la zona no saturada. EVT: evapotranspiración; PFCC: plano flujo cero convergente; PFCD: plano flujo cero divergente; n.f.: nivel freático

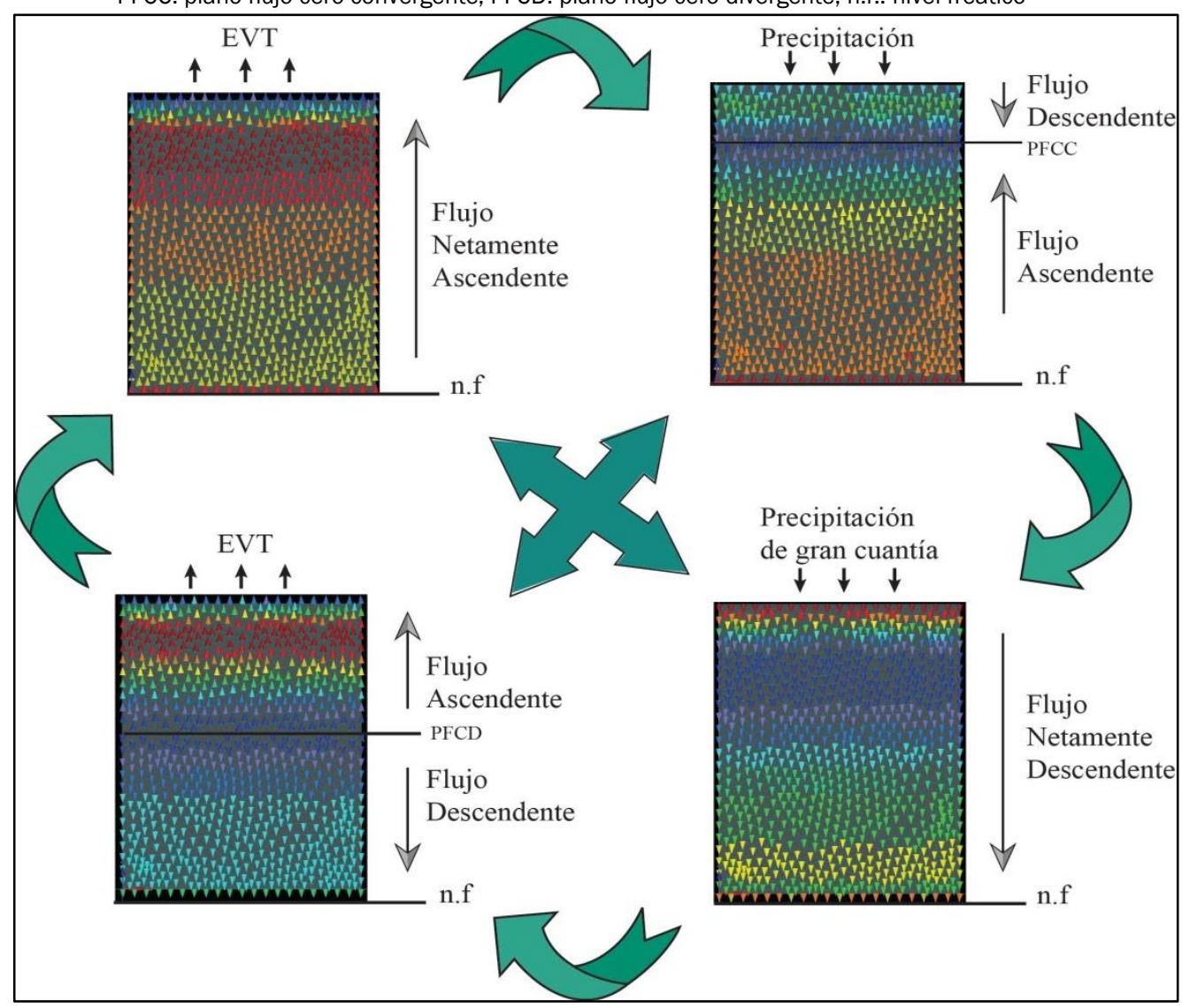

\subsection{Evaluación de la movilidad de sales solubles}

La movilidad de las sales solubles en la zona no saturada (ZNS) fue simulada a partir de considerar a las mismas como soluto no reactivo, siendo los procesos de advección y dispersión hidrodinámica los encargados del transporte. El acuífero freático constituye la fuente de salinidad que se incorpora a la ZNS a partir de los procesos explicados anteriormente.

Las concentraciones salinas en el suelo, se encuentran ligadas directamente a los flujos hídricos no saturados. Los nodos de $15 \mathrm{~cm}$ y $30 \mathrm{~cm}$ reflejan la movilidad de sales solubles en los niveles superficiales del suelo. Durante periodos de escasas precipitaciones (menor a $5 \mathrm{~mm}$ acumulados en días suce- sivos) y gran demanda de humedad atmosférica (meses de verano), priman los movimientos de humedad verticales ascendentes generando la acumulación de sales en los niveles superficiales del suelo por evapotranspiración. En estos periodos las concentraciones salinas presentan picos de hasta 18000 mg.L-1, siendo muy superiores a las concentraciones halladas en el acuífero freático (Fig. 7). Los efectos pueden verse magnificados especialmente durante periodos de stress hídrico, como los ocurridos en los meses de verano del año 2013 y 2015. Esta condición puede observarse en la figura 8a, correspondiente al mes de diciembre del año 2013, momento donde se registran altas tasas de evapotranspiración y lluvias nulas durante 47 días desde el mes de noviembre (Fig. 7). 
Figura 7 - Simulación de las concentraciones salinas para los niveles superficiales del suelo. En la parte superior se observan los flujos atmosféricos (precipitación y evapotranspiración), indicándose con flechas los periodos más relevantes de acumulación y lavado y dilución.

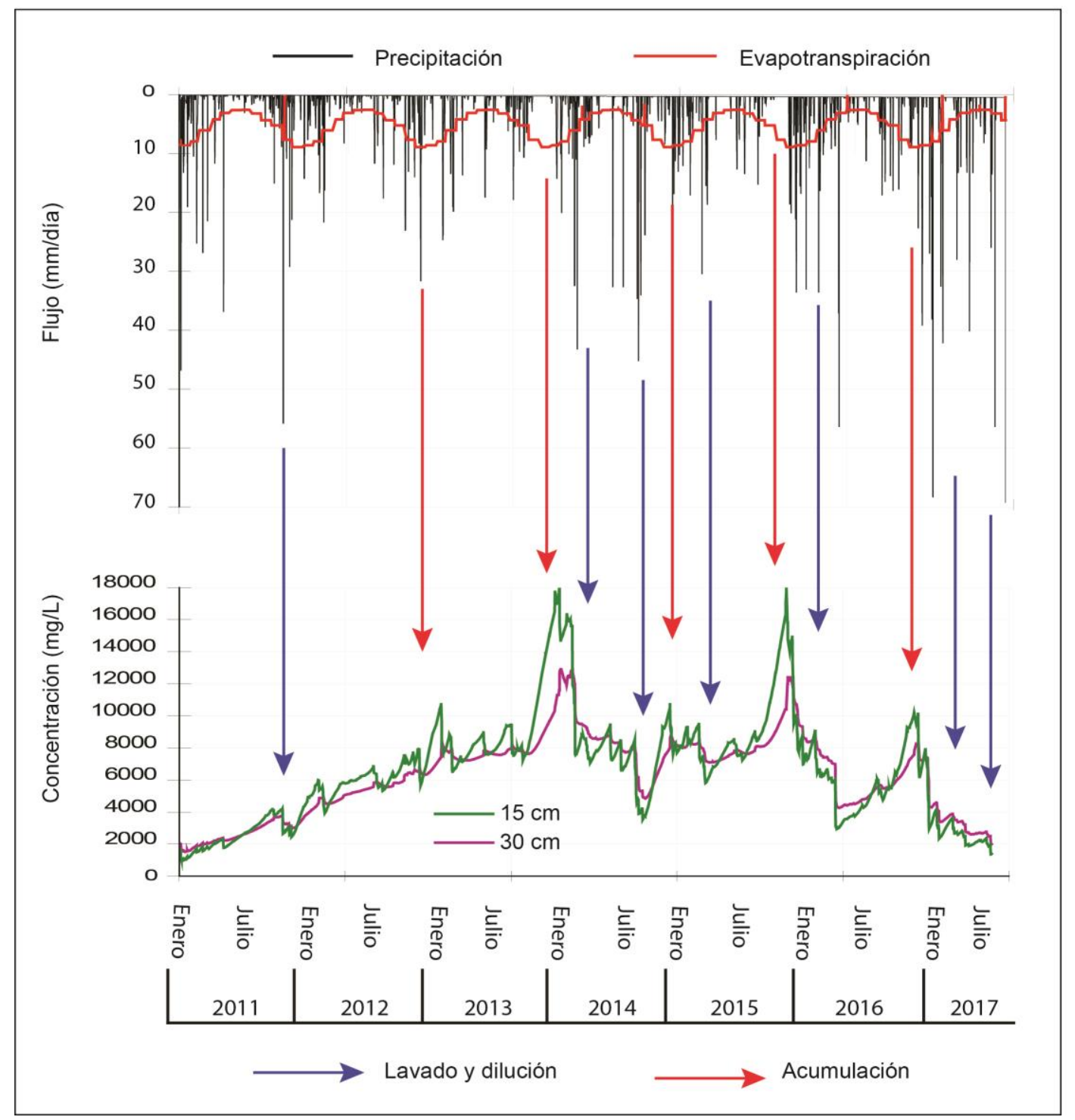

Las precipitaciones aportan agua de baja salinidad al suelo, promoviendo los procesos de lavado y dilución. Este efecto es observable en años húmedos como el 2011, 2014, 2016 y 2017, donde las precipitaciones superaron los $500 \mathrm{~mm}$ anuales. Durante los meses lluviosos el lavado remueve las sales de los niveles superficiales y transporta los solutos hacia la base del perfil, efecto registrado por ejemplo en el mes de septiembre de 2011 (Fig. 8b), luego de transcurrida una precipitación de $56 \mathrm{~mm}$. El perfil de suelo registra concentraciones cercanas a $2000 \mathrm{mg} . \mathrm{L}^{-1}$ en los niveles superficiales incrementándose hasta 5000 mg.L-1 en la base. 
Figura 8- Perfil de concentración salina vs contenidos volumétricos de humedad. A) Periodos donde prima la acumulación. B) Periodos donde ocurren el lavado y dilución. n.f: nivel freático

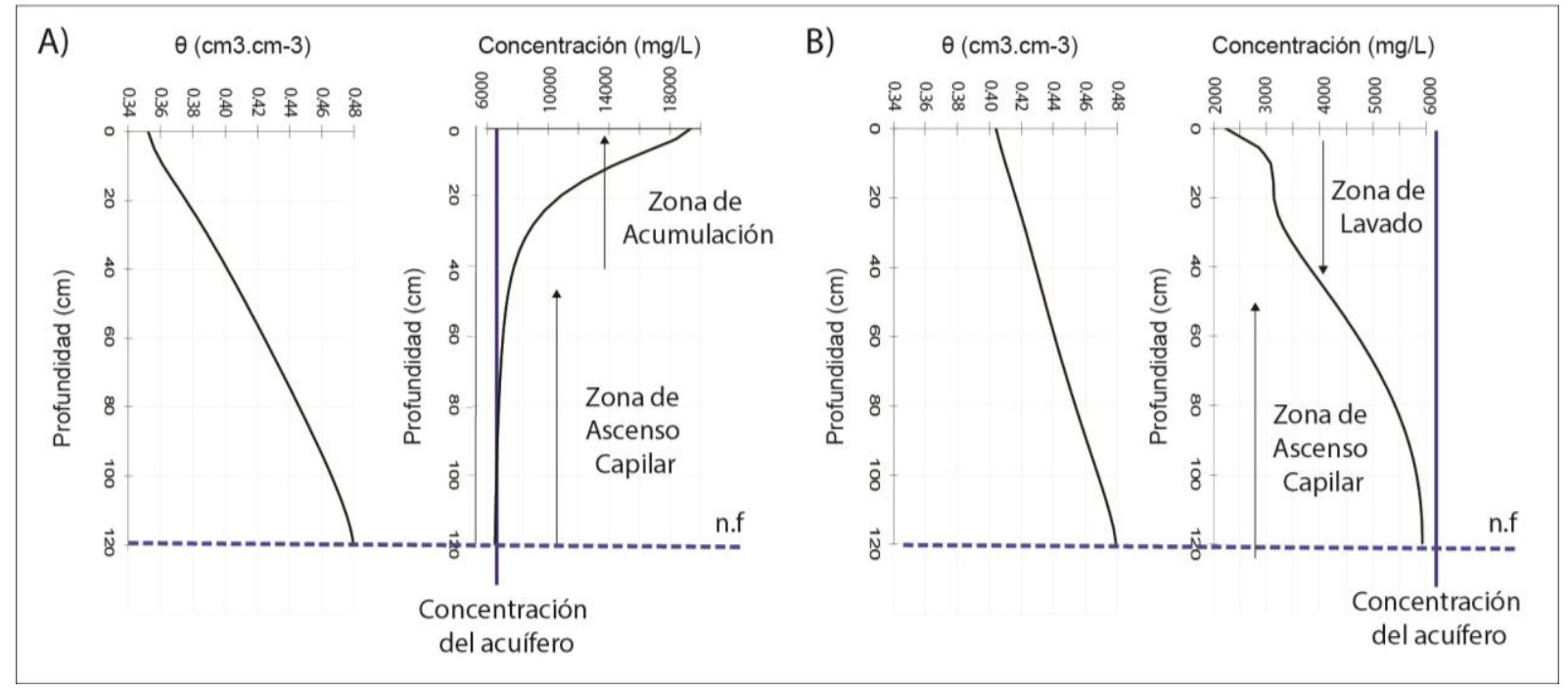

En sectores profundos de la sección, la posición del nivel freático ejerce influencia sobre las concentraciones salinas del mismo modo que lo hace sobre la hidrodinámica. Los nodos de $60 \mathrm{~cm}$ y $90 \mathrm{~cm}$ reflejan las concentraciones encontradas en la franja capilar; cabe destacar que a estas profundidades las tensiones matriciales se mantuvieron por debajo del valor de tensión de entrada de aire $\left(h_{a}\right)$ para todo el periodo simulado (Fig. 4). En esta zona las concentraciones se mantuvieron en el rango de entre 3000 mg.L-1 y 10000 mg.L-1 en pseudoequilibrio a la concentración del acuífero freático (6400 mg.L-1)

(Fig. 9). A diferencia de las concentraciones simuladas para los niveles superficiales, los nodos de $60 \mathrm{~cm}$ y $90 \mathrm{~cm}$ reflejan sus picos de concentración durante periodos de lluvia ocurridos inmediatamente luego de intervalos de tiempo donde predomino la acumulación de sales en superficie (meses de verano cálidos y secos). Este efecto, observable en los meses de febrero y marzo de 2014 y 2016 respectivamente, es explicado por el transporte de las sales presentes en los niveles superficiales del suelo hacia la base del perfil.

Figura 9 - Simulación de las concentraciones salinas para los niveles inferiores del perfil. En la parte superior se indican las precipitaciones (mm/día) para el periodo FC: Franja Capilar

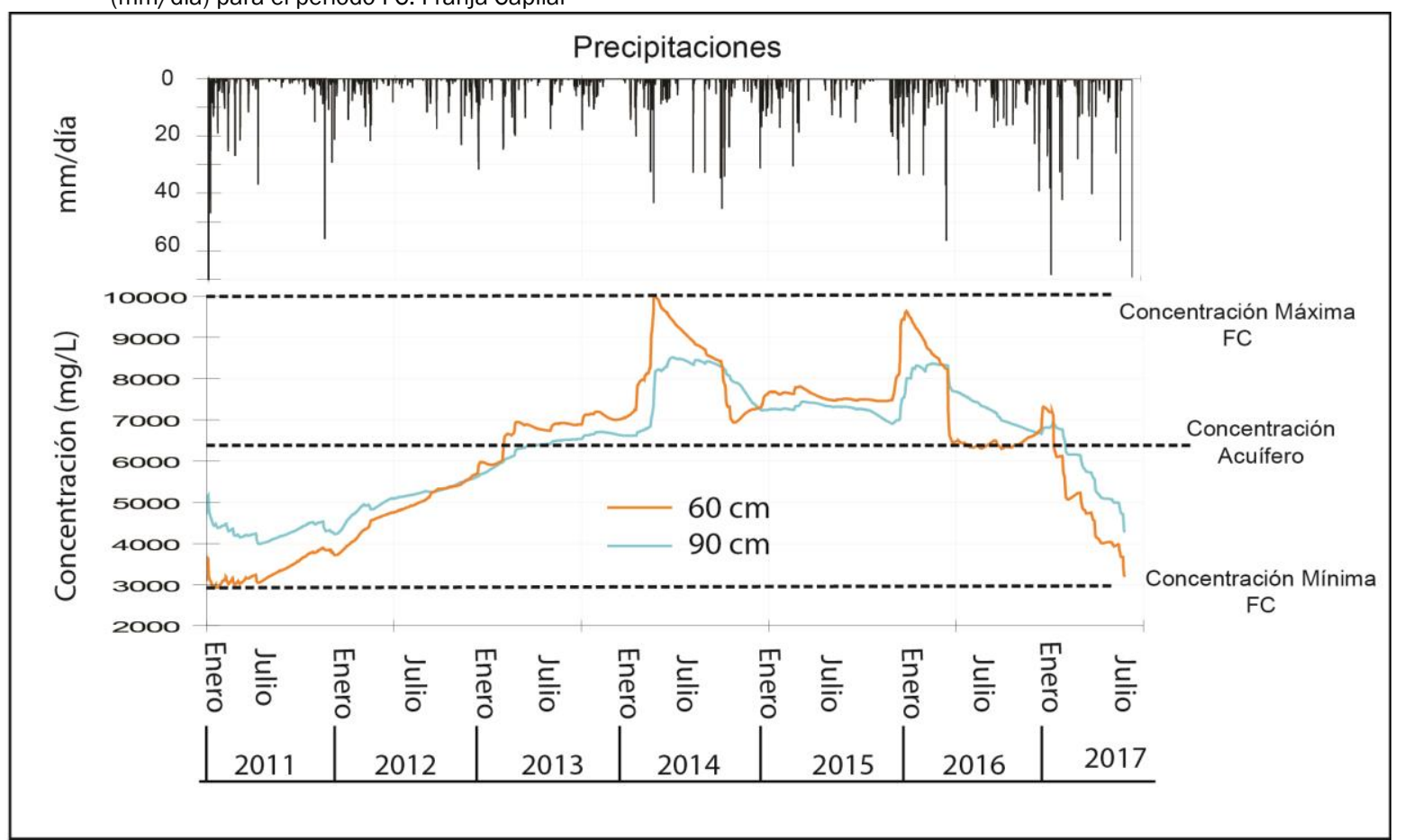


El modelo propuesto para el trasporte de sales solubles en el perfil del suelo se ajusto a la situación actual de campo, correspondiente a las concentraciones medias para los suelos analizados durante el mes de septiembre de 2017 (Fig. 10). De este modo, se verifica que la utilización de datos hidrológicos e hidroquímicos antecedentes como condiciones iniciales del modelo, es factible para tiempos prolongados de simulación. El código Hydrus representa un instrumento útil para la simulación del transporte de sales solubles, en los casos que se dispongan datos de campo únicamente para un rango de tiempo restringido.

Figura 10 - Comparación de las concentraciones salinas en el perfil no saturado simulado vs medido para el tiempo final de simulación.

\section{CONCLUSIONES}

Las simulaciones con el código HYDRUS 2D/3D permitieron modelar la dinámica salina, lográndose distinguir la influencia de la franja capilar y de las condiciones atmosféricas sobre las concentraciones de sales en el perfil de suelo. Los periodos cálidos y secos comprenden el escenario de mayor peligro para los pavimentos y estructuras de hormigón en el sector Sudoeste de la ciudad de Bahía Blanca.

Los periodos de mayor acumulación de sales corresponden a los meses de verano (diciembre, enero y febrero) de la serie de datos en estudio. En este periodo predominan los movimientos verticales ascendentes de humedad dentro de la zona no saturada, sobre los movimientos descendentes, especialmente dada la ocurrencia de precipitaciones escasas a nulas durante estos meses. En estas condiciones se generan concentraciones de sales en los niveles superficiales del suelo de hasta $18000 \mathrm{mg} \cdot \mathrm{L}^{-1}$.

Los suelos resultan con problemas de salinidad asociados a la transferencia de solutos desde el acuífero somero e hipersalino a partir del ascenso capilar, el cual es puesto en marcha por el proceso de evapotranspiración y favorecido por la textura fina de los sedimentos que componen la zona no saturada.

Las precipitaciones permiten el ingreso de agua de baja salinidad al perfil de suelo, ocasionando los procesos de lavado y dilución. Este efecto es recurrente en años húmedos como el 2011, 2014, 2016 y 2017, donde las precipitaciones superaron los $500 \mathrm{~mm}$ anuales. Las sales son transportadas hacia los niveles inferiores de la zona no saturada. Las concentraciones simuladas en la franja capilar se mantuvieron en el rango de entre 10000 mg.L-1 y los 3000 mg.L-1 en pseudoequilibrio a la concentración del acuífero freático (6400 mg.L-1). Las mayores concentraciones de sales en dicha zona se registran en periodos de lluvia ocurridos luego de intervalos de tiempo donde predomino la acumulación de sales en superficie (meses de verano cálidos y secos).

El modelo propuesto para el trasporte de sales solubles permitió arribar a condiciones similares a las actuales de campo. Se verifica que la utilización de datos hidrológicos e hidroquímicos antecedentes como condiciones iniciales del modelo, es factible para la aplicación del código Hydrus en tiempos prolongados de simulación.

Conocer el proceso de salinización de suelos en áreas urbanas, será fundamental para mitigar las consecuencias de las zonas afectadas, soluciones que podrán hacerse extensivas a regiones con problemáticas similares.

\section{REFERENCIAS}

BONORINO, A. G. Y SALA, J. M. Geohidrología. Comisión Estudio de Suelos White-Cerri. MOP de la Provincia de Buenos Aires. Informe Final. La Plata. Inédito, 1983.

CARRICA, J. Hidrogeología de la Cuenca del Arroyo Napostá Grande, Provincia de Buenos Aires. Tesis (Doctoral)- Universidad Nacional del Sur. Biblioteca Central, 215pp y anexos. Inédito, 1998. 
CALÓ, J., E. FERNÁNDEZ, A. MARCOS Y M. SEQUEIRA. Medidas de mitigación de los impactos Ambientales producidos por lluvias intensas en la ciudad de Bahía Blanca. In: SIMPOSIO DE GEOLOGÍA APLICADA A LA INGENIERÍA Y AL MEDIOAMBIENTE, 8., 2004. Anais... Córdoba, Argentina, 2004.

CORWIN D. L., RHOADES, J.D. Y SIMUNEK, J. Leaching requirement for soil salinity control: Steady-state versus transient models. Agricultural Water Management, v. 90, p.165-180, 2007.

FAO. Crop evapotranspiration.Guidelines for computing crop water requirements: FAO Irrigation and drainage: paper 56. FAO - Food and Agriculture Organization of the United Nations. Rome, 1998.

FEDDES, R. A., KOWALIK P. J., AND ZARADNY, H. Simulation of field water use and crop yield. John Wiley \& Sons, New York, NY, 1978.

FIDALGO, F. Geología y Geomorfología del área de White-Cerri y los alrededores de bahía blanca. Comisión Estudio de Suelos White-Cerri. MOP de la provincia de buenos Aires. Inédit, 1983.

FORKUTSA, I., SOMMER, R., SHIROKOVA, I., LAMERS, J.P.A., KIENZLER, K. TISCHBEIN, B., MARTIUS, C. Y VLEK, P.L.G. Modeling irrigated cotton with shallow groundwater in the Aral Sea Basin of Uzbekistan: II. Soil salinity dynamics. Irrigation Science, v.27, p. 319-330, 2009.

GRIZINIK, M. Y HIRTZ, N. Salinización en el ejido urbano de la ciudad de Comodoro Rivadavia, Chubut, Argentina. Evaluación y Propuesta de Saneamiento. In: I CONGRESSO MUNDIAL INTEGRADO DE ÁGUAS SUBTERRÂNEAS, 1., 2000. Anais... Fortaleza. São Paulo: ABAS, 2000.

LEXOW, C., G. PERA VALLEJOS Y E. BAUER. Comportamiento de la franja capilar en el sector oeste de la Ciudad de Bahía
Blanca. Actas XX Congreso Geológico Argentino, Sesión Técnica 15, p. 65-76, 2017.

MUALEM, Y. A new model for predicting the hydraulic conductivity of unsaturated porous media. Water Resources Research, v.12, n. 3, p. 513-522, 1976.

PHOGAT, V., SKEWES, M.A., COX, J.W., SANDERSON, G., ADAM J. Y SIMUNEK, J. Seasonal simulation of water, salinity and nitrate dynamics under drip irrigated mandarin (Citrus reticulata) and assessing management options for drainage and nitrate leaching. Journal of Hydrology, v. 513, p. 504-516, 2014.

SCHAAP, M. G., LEIJ, F.J. AND VAN GENUCHTEN, M. TH. Rosetta: a computer program for estimating soil hydraulic parameters with hierarchical pedotransfer functions. Journal of Hydrology, v. 251, p. 163-176, 2001.

SIMUNEK, J., SEJNA M., AND VAN GENUCHTEN M. TH. The HYDRUS (2D/3D) software package for simulating the two- and three-dimensional movement of water, heat and multiple solutes in variably saturated media. Version 1.0.PC Progress, Prague, Czech Republic, 2006.

USDA (UNITED STATED DEPARTMENT OF AGRICULTURE), Keys to Soil Taxonomy. Natural Resources Conservation Service (NRCS). Ninth Edition, 2003. 332 p.

VAN GENUCHTEN, M. TH. A closed-form equation for predicting the hydraulic conductivity of unsaturated soils. Soil Science Society of American Journal, v. 44, n. 3, p.892-898, 1980.

WESSELING, J.G. Simulación multianual del flujo de agua subterránea para diferentes perfiles de suelo, niveles de agua subterránea y cultivos con el modelo SWATRE. Wageningen, DLO-Staring Centrum, Repport 152, 1991.63p. 